\title{
On Systems of Difference Equations with Wrong Boundary Conditions*
}

\author{
By Stanley Osher
}

\begin{abstract}
Kreiss and Lundquist [1], have recently obtained certain results about the decay of influence of wrong boundary conditions in overdetermined difference approximations to a hyperbolic partial differential equation. We have extended this result to include systems of hyperbolic partial differential equations in several space variables and their corresponding overdetermined difference approximations.

The technique of proof involves examining sections of the resolvent of the difference operator for analyticity within the unit circle as in Kreiss [6] and Osher [2].
\end{abstract}

I. Introduction. In this paper we shall generalize some results of Kreiss and Lundquist [1] to the system

$$
u_{t}=A u_{x}+\sum_{i=1}^{m} B_{i} u_{y i}, \quad y=\left[y_{1}, \cdots, y_{m}\right]
$$

to be solved for the $n$ vector $u(x, y, t)$ in $0 \leqq x, t>0,-\infty<y_{i}<\infty, i=1,2$, $\cdots, m$.

$A$ and the $B_{i}$ are each constant $n \times n$ matrices. We assume that the system is hyperbolic, i.e. for arbitrary real values of $\xi$ and $\eta_{j}$, the matrix

$$
\xi A+\sum_{j=1}^{m} \eta_{j} B_{j}
$$

has only real eigenvalues $\tau$.

We make the important assumption:

(A1) The eigenvalues $d_{i}$ which obey $\operatorname{det}\left[A-d_{i}\right]=0, i=1,2, \cdots, n$, are such that $d_{i}>0, i=1,2, \cdots, n$.

This means that $x=0$ is noncharacteristic and that the curves $t=\phi(x)$ formed by the intersection of a characteristic surface and a plane $y=$ constant have the property that $\phi^{\prime}(x)<0$. (These bicharacteristics go into the boundary from the region.)

In [1], the equation

$$
u_{t}=u_{x}, \quad x, t>0,
$$

was discussed. The proof of the main result in [1] does not go over to our case essentially because we shall be dealing with a difference operator whose $l_{2}$ norm need not equal its spectral radius, unlike the scalar Toeplitz operators of Strang [3].

We approximate (1.1) by a difference equation of the form:

$$
\begin{aligned}
& v\left(x, y_{1}, \cdots, y_{m}, t+k\right) \\
& \quad=\sum_{j=-s}^{l} \sum_{j_{1}=-s_{1}}^{l_{1}} \cdots \sum_{j_{m}=-s_{m}}^{l_{m}} C_{j, j_{1}, \cdots, j_{m}} v\left(x+j h, y_{1}+j_{1} h_{1}, \cdots, y_{m}+j_{m} h_{m}, t\right),
\end{aligned}
$$

Received June 3, 1968, revised November 14, 1968.

* Research carried out at Brookhaven National Laboratory under contract with the U. S. Atomic Energy Commission. 
$x=p h \geqq 0, y_{i}=p^{i} h_{i}, t=q k$, for $q, p=1,2, \cdots, p^{i}=\cdots,-1,0,1, \cdots$, $h>0$ and $h / k=\mu, h_{i} / k=\mu_{i}>0$ for $\mu, \mu_{i}$ fixed as $k \rightarrow 0$.

For both (1.1) and (1.4) we choose initial conditions:

$$
v\left(x, y_{1}, \cdots, y_{m}, 0\right)=u\left(x, y_{1}, \cdots, y_{m}, 0\right)=0, \quad x>0 \text { and all } y_{i} .
$$

We make the assumption:

(A2) (1.4) is consistent with (1.1).

The main theorem of Hersh [4] assures us that the problem (1.1) with initial conditions is well posed, and hence (1.5) implies that the solution is $u\left(x, y_{1}, \cdots, y_{m}, t\right)$ $\equiv 0$. However we need additional data in order to use (1.4), i.e. we need $v\left(x, y_{1}, \cdots, y_{m}, t\right)$ for $(1-s) h \leqq x \leqq 0$. We introduce extra boundary conditions:

$$
v\left(x, y_{1}, \cdots, y_{m}, t\right)=g\left(x, y_{1}, \cdots, y_{m}, t\right)
$$

for $h-s h \leqq x \leqq 0$, where $g$ is square integrable in each $y_{i}$ and $\exists M>0$ such that

$$
\sup _{x, t} \int_{y_{m}=-\infty}^{\infty} \cdots \int_{y_{1}=-\infty}^{\infty}\left|g\left(x, y_{1}, \cdots, y_{m}, t\right)\right|^{2} d y_{1} \cdots d y_{m}<M .
$$

(The absolute value of an $n$-vector is the square root of the sum of the squares of its components.)

Consider the symbol of the difference operator:

$$
\begin{aligned}
C\left(e^{i \theta}, e^{i \xi_{1}}, \cdots, e^{i \xi_{m}}\right) \\
\quad=\sum_{j=-s}^{l} \sum_{j_{1}=-s_{1}}^{l_{1}} \cdots \sum_{j_{m}=-s_{m}}^{l_{m}} C_{j, j_{1}, \cdots, j_{m}} \exp \left[i\left(j \theta+j_{1} \xi_{1}+\cdots+j_{m} \xi_{m}\right)\right] .
\end{aligned}
$$

We make the assumptions:

(A3) For all real $\theta, \xi_{1}, \cdots, \xi_{m},|\theta| \leqq \pi$, each $\left|\xi_{j}\right| \leqq \pi$, the eigenvalues $\lambda_{i}\left(e^{i \theta}, \cdots, e^{i \xi m}\right)$ satisfy

$$
\left|\lambda_{i}\left(e^{i \theta}, \cdots, e^{i \xi_{m}}\right)\right| \leqq 1
$$

(A4) For all real $\theta, \xi_{1}, \cdots, \xi_{m}$ with $|\theta| \leqq \pi,\left|\xi_{i}\right| \leqq \pi, i=1,2, \cdots, m$, and some $\alpha>0$, there is a $\beta>0$ such that

$$
\left|\lambda_{i}\left(e^{i(\theta+i \alpha)}, e^{i \xi_{1}}, \cdots, e^{i \xi_{m}}\right)\right| \leqq e^{-\beta} .
$$

A difference scheme having the last two properties is called contractive.

Now we define

$$
\sum_{j_{m}=-s_{m}}^{l_{m}} \ldots \sum_{j_{1}=-s_{1}}^{l_{1}} C_{j, j_{1}, \cdots, j_{m}} \exp \left[-i\left(j_{1} \xi_{1}+\cdots+j_{m} \xi_{m}\right)\right]=C_{j}\left(e^{i \xi}\right) .
$$

Fix $\xi=\left[\xi_{1}, \cdots, \xi_{m}\right]$ and consider the operator $\hat{T}(\xi)$ defined by

$$
\omega_{k}=(\hat{T}(\xi) \hat{f})_{k}=\sum_{j=\max }^{l} \sum_{((1-s-k),-s)} C_{j}\left(e^{i \xi}\right) f_{j+k}, \quad k=1-s, 2-s, \cdots,
$$

on the $l_{2}$ space of sequences

$$
\hat{\omega}=\left\{\omega_{-s+1}, \cdots, \omega_{0}, \cdots\right\} .
$$

We make the assumption: 
(A5) For any real $\xi$ and for $|\lambda| \geqq 1$, it is true that

$$
[\hat{T}(\xi)-\lambda] \hat{f}=0 \Leftrightarrow \hat{f}=0 .
$$

Finally we assume for all real $\xi$

$$
\begin{aligned}
& \operatorname{det}\left[C_{-s}\left(e^{i \xi}\right)\right] \neq 0, \\
& \operatorname{det}\left[C_{l}\left(e^{i \xi}\right)\right] \neq 0 .
\end{aligned}
$$

We may now state our main result.

Theorem. Let all five assumptions be valid. It then follows that the solution to (1.4), (1.5) obeys

$$
\left(\int_{-\infty}^{\infty} \ldots \int_{-\infty}^{\infty}\left|v\left(x, y_{1}, \cdots, y_{m}, t\right)\right|^{2} d y_{1} \cdots d y_{m}\right)^{1 / 2} \leqq k_{2} e^{-x / h \alpha} M
$$

for some constant $k_{2}$.

This result implies that the influence of the inhomogeneous boundary conditions is present in an interval $\approx$ const $h|\log h|$.

The following lemma assures us that assumption (A4) is valid for the difference schemes ordinarily used to approximate Eq. (1.1).

Lemma 1. Let the eigenvalues of the symbol (1.8) be strictly less than one in absolute value for real $\theta$ and $\xi$ unless $0=\theta=\xi_{1}=\xi_{2} \cdots=\xi_{m}$. Let $A$ be a diagonal matrix. Then assumption (A4) is valid.

II. Proof of Theorem I. For any fixed $t, k, h$, we consider the space $\left(L_{2}\right)^{m+1}$ of grid-functions with $v(t)=v\left(x, y_{1}, \cdots, y_{m}, t\right)$ and

$$
\begin{aligned}
\left\|v\left(x, y_{1}, \cdots, y_{m}, t\right)\right\|^{2} & \\
& =h h_{1} \cdots h_{m} \sum_{p=-s+1}^{\infty} \sum_{p^{1}=-\infty}^{\infty} \cdots \sum_{p^{m}=-\infty}^{\infty}\left|v\left(p h, p^{1} h_{1}, \cdots, p^{m} h_{m}, t\right)\right|^{2} .
\end{aligned}
$$

On this space, we may replace (1.4), (1.5) and (1.6) by

$$
v(t+k)=P g(t+k)+(I-P) T v(t),
$$

where $P$ is the orthogonal projector defined by

$$
\begin{aligned}
& \left(P\left(v\left(x, y_{1}, \cdots, y_{m}, t\right)\right)\right)_{(x)} \equiv 0 \quad \text { if } x>0, \\
& \left(P\left(v\left(x, y_{1}, \cdots, y_{m}, t\right)\right)\right)_{(x)} \equiv v\left(x, y_{1}, \cdots, y_{m}, t\right) \quad \text { if } x \leqq 0,
\end{aligned}
$$

and $T$ is the Toeplitz-convolution operator defined by

$$
\begin{aligned}
& \omega\left(x, y_{1}, \cdots, y_{m}, t\right)=T v \\
&=\sum_{j=\max } \sum_{(-s, 1-s-x / h)}^{l} \sum_{j_{1}=-s_{1}}^{l_{1}} \ldots \sum_{j_{m}=-s_{m}}^{l_{m}} C_{j, j, j_{2}, \cdots, j_{m}} \\
& \cdot v\left(x+j h, y_{1}+j_{1} h_{1}, \cdots, y_{m}+j_{m} h_{m}, t\right) .
\end{aligned}
$$

It thus follows that

$$
v(t)=P g(t)+\sum_{j=1}^{((t-1) / k)}((I-P) T)^{(t / k-j)} P g(j k) .
$$

If $k_{0} \geqq 1$, then 


$$
((I-P) T)^{k_{0}}=((I-P) T(I-P))^{k_{0}-1}(I-P) T P .
$$

We may use

$$
(\lambda I-(I-P) T(I-P))^{-1}=\sum_{j=0}^{\infty} \frac{((I-P) T(I-P))^{j}}{\lambda^{j+1}},
$$

for $|\lambda|$ sufficiently large, as a generating function for the powers of $(I-P) T(I-P)$.

We next take the Fourier transform with respect to the $y$ variables:

$$
\begin{aligned}
\hat{v}(t) & =\hat{v}\left(x, e^{i \xi_{1}}, \cdots, e^{i \xi_{m}}, t\right) \\
& =\sum_{p^{1}=-\infty}^{\infty} \cdots \sum_{p^{m=-\infty}}^{\infty} v\left(p h, p^{1} h_{1}, \cdots, p^{m} h_{m}, t\right) e^{i\left(p^{1} \xi_{1}+\cdots+p^{m} \xi_{m}\right)}
\end{aligned}
$$

and use the obvious integral norm on $|\xi| \leqq \pi$ which creates an isometry.

On the Fourier space $\hat{L}_{2},(2.4)$ becomes

$$
\hat{\omega}\left(x, e^{i \xi}\right)=\hat{T} v=\sum_{j=\max } \sum_{(-s, 1-s-x / h)}^{l} C_{j}\left(e^{i \xi}\right) \hat{v}\left(x+j h, e^{i \xi}\right) .
$$

Consider the orthogonal projections on $\hat{L}_{2}$

$$
\begin{aligned}
& Q_{j h}\left(\hat{v}\left(x, e^{i \xi}\right)\right)=v\left(j h, e^{i \xi}\right) \quad \text { if } x=j h, \\
& Q_{j h}\left(\hat{v}\left(x, e^{i \xi}\right)\right)=0 \quad \text { if } x \neq j h .
\end{aligned}
$$

From (2.5), it is clear that we must prove

$$
\left\|Q_{\nu h} \sum_{j=1}^{t / k}((I-\hat{P}) \hat{T})^{t / k-j} \hat{P} \hat{g}(j k)\right\| \leqq k_{2} e^{-\alpha \nu} M
$$

for $\nu=0,1, \cdots$.

We may easily show that

$$
(\lambda I-(I-\hat{P}) \hat{T}(I-\hat{P}))^{-1}=\hat{P} / \lambda+(I-\hat{P})(\lambda-\hat{T})^{-1}(I-\hat{P}) .
$$

We are thus merely concerned with

$$
\begin{aligned}
\| \frac{Q_{\nu h}}{2 \pi i} \sum_{j=1}^{t / k-2} \underset{|\lambda|=1+\epsilon}{\Phi} d \lambda \lambda^{t / k-j}(I-\hat{P})(\lambda-\hat{T})^{-1}(I-\hat{P}) T \hat{P} g(j k) \\
+Q_{\nu h}(I-\hat{P}) \hat{T} \hat{P} \hat{g}(t k)+Q_{\nu h} \hat{P} \hat{g}(t) \|
\end{aligned}
$$

for $\epsilon>0$.

It is clear that if $\nu \geqq s+1$, then

$$
Q_{\nu h}(I-\hat{P}) \hat{T} \hat{P} \hat{g}(t-k)=Q_{\nu h} \hat{P} \hat{g}(t)=0 .
$$

We must thus merely estimate

$$
\left\|Q_{\nu h} \sum_{j=1}^{t / k-2} \underset{|\lambda|=1+\epsilon}{\Phi} d \lambda \lambda^{t / k-j}(I-\hat{P})(\lambda-\hat{T})^{-1}(I-\hat{P}) \hat{T} \hat{P} g(\jmath k)\right\| .
$$

We may also easily show that it is sufficient to estimate 


$$
\left\|Q_{\nu h} \sum_{j=1}^{t / k-2} \underset{|\lambda|=1+\epsilon}{\oint} d \lambda \lambda^{t / k-j}(\lambda-\hat{T})^{-1} \hat{P} \hat{f}(j k)\right\|
$$

where $\|\hat{f}(j k)\| \leqq\|T\|\|\hat{g}(j k)\|$.

Kreiss [6] has obtained the resolvent of $T$. We use formula (2.10), page 709, [6]. Thus, conditions (A3), (A5), and (A6) guarantee the existence of this resolvent if $|\lambda|>1$. We thus know

$$
(\hat{T}(\xi)-\lambda) \hat{w}(\xi)=\hat{v}(\xi),
$$

where $\hat{v}(x, \xi)=0$ for $x \geqq 0$, has the solution

$$
\hat{w}(j h, \xi)=\sum_{p=1-s}^{\min (j, 0)} M_{(j, p)}(\xi, \lambda) \hat{v}(p h, \xi)
$$

for $|\lambda|>1$ and all real $\xi$. Moreover, the matrices $M_{(j, p)}(\xi, \lambda)$ are analytic in all their variables as long as

(1) the roots of the polynomial

$$
\operatorname{det}\left[C_{l}(\xi) z^{l+s}+\cdots+\left(C_{0}(\xi)-\lambda\right) z^{s}+\cdots+C_{-s}(\xi)\right]=0
$$

have the property that $s n$ of them lie within some circle $|z|<R_{1}$ and $\ln$ of them lie in the region $R_{1}<|z|$,

(2) a certain determinant $[F(\lambda, \xi)] \neq 0$.

Condition (A5) guarantees that (2) is true for $|\lambda| \geqq e^{-\gamma_{1}, \beta}>\gamma_{1}>0$. Condition (A4) guarantees that (1) is true for $|\lambda|>e^{-\beta}$, and in fact $R_{1}=e^{-\alpha}$. Finally, in this region, it is true that

$$
\left\|M_{(j, p)}(\xi, \lambda)\right\| \leqq k_{3} R_{1}^{j-p}=k_{3} e^{-\alpha(j-p)} .
$$

Thus, from the Cauchy integral formula, we have

$$
\begin{aligned}
\left\|Q_{\nu h} \underset{|\lambda|=1+\epsilon}{\Phi} d \lambda \lambda^{t / k-j}(I-P)(\lambda-T)^{-1}(I-P) T P\right\| & \\
& \leqq k_{4} e^{-\nu \alpha} \exp \left[-\gamma_{1}(t / k-j)\right]
\end{aligned}
$$

and

$(2.22)\left\|Q_{\nu h} \sum_{j=1}^{t / k-2} \underset{|\lambda|=1+\epsilon}{\Phi} d \lambda \lambda^{t / k-j}(I-P)(\lambda-T)^{-1}(I-P) T P\right\| \leqq k_{5} e^{-\nu \alpha}$. Q.E.D.

III. Proof of Lemma I. Consider the solutions to (2.16). If $|\lambda| \geqq 1, \lambda \neq 1$ or $\lambda=1$ and some $\xi_{i} \neq 0$, then sn of the solutions are within the unit circle, $\ln$ are outside the unit circle. Consistency assures us that exactly $n$ of the zeros approach 1 as $\lambda \rightarrow 1$ and $\xi_{1} \rightarrow 0, \cdots, \xi_{m} \rightarrow 0$. Consistency and the fact that $A$ is diagonal and positive-definite imply that these $n$ were originally outside the unit circle. Thus the $s n$ solutions which were originally within the unit circle actually remain within some smaller circle $|z|<e^{-\alpha}$ for $|\lambda| \geqq e^{-\beta}$ and the other $\ln$ solutions remain outside this circle for such $\lambda$. This implies assumption (A4).

The author would like to thank the referee for helping to make the paper more compact. 
University of California

Department of Mathematics

Berkeley, California 94720

1. H. O. Kreiss \& E. Lundquist, "On difference approximations with wrong boundary values," Math. Comp., v. 22, 1968, pp. 1-12.

2. S. OsHER, "Systems of difference equations with general homogeneous boundary conditions," Trans. Amer. Math. Soc., v. 137, 1969, pp. 177-202.

3. G. W. STRANG, "Wiener-Hopf difference equations," J. Math. Mech., v. 13, 1964, pp. 85-96. MR $28 \# 3548$.

4. R. Hersh, "Mixed problems in several variables," J. Math. Mech., v. 12, 1963, pp. 317334. MR $26 \# 5304$.

5. S. V. PARTER, "Stability, convergence and pseudo-stability of finite-difference equations for an over-determined problem," Numer. Math., v. 4, 1962, pp. 277-292. MR 26 \#5740.

6. H.-O. KREISS, "Stability theory for difference approximations of mixed initial boundary value problems. I," Math. Comp., v. 22, 1968, pp. 703-714. 